\title{
Thanks to all those who reviewed for Trials in 2014
}

Douglas G Altman ${ }^{1 *}$, Curt D Furberg ${ }^{2}$ and Jeremy M Grimshaw ${ }^{3}$

\section{Contributing reviewers}

A peer-reviewed journal would not survive without the generous time and insightful comments of the reviewers, whose efforts often go unrecognized. Although final decisions are always editorial, they are greatly facilitated by the deeper technical knowledge, scientific insights, understanding of social consequences, and passion that reviewers bring to our deliberations. For these reasons, the Editors-in-Chief and staff of the journal warmly thank the 610 reviewers whose comments helped to shape Trials, for their invaluable assistance with review of manuscripts for the journal in Volume 15 (2014).

Purva Abhyankar

UK

Hatem Abu Hashim

Egypt

Clive E Adams

UK

Joy Adamson

UK

Christopher Adlbrecht

Austria

Luciano Agati

Italy

Rakesh Aggarwal

India

Mahboob Alam

USA

Eric Albrecht

Switzerland

\author{
Muktar Aliyu \\ USA
}

Kelli Allen

USA

Osvaldo Almeida

Australia

Constantine Amditis

Australia

Gavin Andrews

Bahamas

Djillali Annane

France

Massimo Antonelli

Italy

Ane Appelt

Denmark

Bruce Arroll

New Zealand
Deborah Ashby

UK

Lisa Askie

Australia

George Atkinson

USA

Peter Bacchetti

USA

Franz G. Bader

Germany

Gillian Baer

UK

Anne-Marie Bagnall

UK

\section{Sean Bagshaw}

Canada

Patricia Ballesta

Spain

\footnotetext{
* Correspondence: doug.altman@csm.ox.ac.uk

${ }^{1}$ Centre for Statistics in Medicine, University of Oxford, Botnar Research

Centre, Windmill Road, Oxford OX3 7LD, UK

${ }^{2}$ Division of Public Health Sciences, Wake Forest University School of

Medicine, Medical Center Boulevard, Winston-Salem, NC 27157-1063, USA

${ }^{3}$ Clinical Epidemiology Programme, Ottawa Hospital Research Institute and

Department of Medicine, University of Ottawa, 501 Smyth Road, Ottawa, ON

K1H 8 L6, Canada
}

\section{Biomed Central}

(c) 2015 Altman et al.; licensee BioMed Central. This is an Open Access article distributed under the terms of the Creative Commons Attribution License (http://creativecommons.org/licenses/by/4.0), which permits unrestricted use, distribution, and reproduction in any medium, provided the original work is properly credited. The Creative Commons Public Domain Dedication waiver (http://creativecommons.org/publicdomain/zero/1.0/) applies to the data made available in this article, unless otherwise stated. 
Karla Ballman

USA

Barbara Bardoni

France

Neil Barnes

UK

Guido Bastiaens

Netherlands

Erika Baum

Germany

Shrujal Baxi

USA

David Beard

UK

Beatrice Beck-Schimmer

Switzerland

Vincenzo Berghella

USA

Julie Bernhardt

Australia

Pietro Bertini

Italy

Martin Bertrand

France

Mireia Besalú

Spain

Zhaoxiang Bian

Hong Kong

José Bilbao

Spain

Ariella Binik

UK

Yvonne Birks

UK

Jonathan Bisson

UK

Steven Blair

USA

Jane Blazeby

UK

Ashley Bonner

Canada

Jonathan Boote

UK
Ron Borland

Australia

Mark Borthwick

UK

Josée Bouchard

Canada

Peter Bower

UK

Mike Bradburn

UK

Patrick Brady

USA

Michael Brainin

Austria

Thijs Brandsma

Netherlands

Christopher Bray

UK

Daniel Bressington

Hong Kong

Laurent Brochard

Canada

Marianne Brodmann

Austria

Gert Bronfort

USA

Richard Brown

USA

Sarah Brown

UK

Sandra Bucci

UK

Stefan Budde

Germany

Lorraine Buis

USA

Bryan Burford

UK

Tom Burns

UK

Martin Burtscher

Austria

Phyllis Butow

Australia
Marc Buyse

Belgium

Agnès Caille

France

Michael Campbell

UK

Bridget Candy

UK

Daniel Carr

USA

Christopher Carroll

UK

Maija Castren

Finland

Cheejen Chang

Taiwan

Xiaorong Chang

China

Sue Channon

UK

Baoying Chen

China

Wei Chen

USA

Yiyi Chen

USA

Christopher Chen

Singapore

Dongquan Chen

USA

Ji Cheng

Canada

Wai Tong Chien

Hong Kong

Pietro Chiurazzi

Italy

Sun-Mi Choi

Korea, South

Babak Choodari-Oskooei UK

Chun-Hung Chu

Hong Kong

Rong Chu

Canada 


\begin{tabular}{|c|c|c|}
\hline $\begin{array}{l}\text { Roberto Cirocchi } \\
\text { Italy }\end{array}$ & $\begin{array}{l}\text { Sara Czaja } \\
\text { USA }\end{array}$ & $\begin{array}{l}\text { Gordon Doig } \\
\text { Australia }\end{array}$ \\
\hline $\begin{array}{l}\text { David Clarke } \\
\text { UK }\end{array}$ & $\begin{array}{l}\text { Dave Dagnan } \\
\text { USA }\end{array}$ & $\begin{array}{l}\text { Tara Donker } \\
\text { Australia }\end{array}$ \\
\hline $\begin{array}{l}\text { Jan Clarkson } \\
\text { UK }\end{array}$ & $\begin{array}{l}\text { Rafael Dal-Ré } \\
\text { Spain }\end{array}$ & $\begin{array}{l}\text { Allan Donner } \\
\text { Canada }\end{array}$ \\
\hline $\begin{array}{l}\text { Elisabeth Coart } \\
\text { Belgium }\end{array}$ & $\begin{array}{l}\text { Sarah Damery } \\
\text { UK }\end{array}$ & $\begin{array}{l}\text { Jenny L. Donovan } \\
\text { UK }\end{array}$ \\
\hline $\begin{array}{l}\text { Erik Cobo } \\
\text { Spain }\end{array}$ & $\begin{array}{l}\text { Antoni Davalos } \\
\text { Spain }\end{array}$ & $\begin{array}{l}\text { Peter Doshi } \\
\text { USA }\end{array}$ \\
\hline $\begin{array}{l}\text { Christopher Coffey } \\
\text { USA }\end{array}$ & $\begin{array}{l}\text { Colin Dayan } \\
\text { UK }\end{array}$ & $\begin{array}{l}\text { George Dowswell } \\
\text { UK }\end{array}$ \\
\hline $\begin{array}{l}\text { Dena Cohen } \\
\text { UK }\end{array}$ & $\begin{array}{l}\text { Sabina De Geest } \\
\text { Switzerland }\end{array}$ & $\begin{array}{l}\text { Rohan D'souza } \\
\text { Canada }\end{array}$ \\
\hline $\begin{array}{l}\text { Judith Cohen } \\
\text { UK }\end{array}$ & $\begin{array}{l}\text { Harry J. De Koning } \\
\text { Netherlands }\end{array}$ & $\begin{array}{l}\text { Joel Dubin } \\
\text { Canada }\end{array}$ \\
\hline $\begin{array}{l}\text { Natasha Cohen } \\
\text { Canada }\end{array}$ & $\begin{array}{l}\text { Jorge De La Torre } \\
\text { USA }\end{array}$ & $\begin{array}{l}\text { Chantale Dumoulin } \\
\text { Canada }\end{array}$ \\
\hline $\begin{array}{l}\text { Ben Colagiuri } \\
\text { Australia }\end{array}$ & $\begin{array}{l}\text { Daniele De Luca } \\
\text { Italy }\end{array}$ & $\begin{array}{l}\text { Graham Dunn } \\
\text { UK }\end{array}$ \\
\hline $\begin{array}{l}\text { Tim Colbourn } \\
\text { UK }\end{array}$ & $\begin{array}{l}\text { Christian De Mey } \\
\text { Germany }\end{array}$ & $\begin{array}{l}\text { David Daniel Ebert } \\
\text { Germany }\end{array}$ \\
\hline $\begin{array}{l}\text { Dave Collett } \\
\text { UK }\end{array}$ & $\begin{array}{l}\text { Jon Deeks } \\
\text { UK }\end{array}$ & $\begin{array}{l}\text { Allison Ross Eckard } \\
\text { USA }\end{array}$ \\
\hline $\begin{array}{l}\text { Deborah Joanne Cook } \\
\text { Canada }\end{array}$ & $\begin{array}{l}\text { Emilia D'elia } \\
\text { Italy }\end{array}$ & $\begin{array}{l}\text { Diana Elbourne } \\
\text { UK }\end{array}$ \\
\hline $\begin{array}{l}\text { Cindy Cooper } \\
\text { UK }\end{array}$ & $\begin{array}{l}\text { Ingrid Demmelmaier } \\
\text { Sweden }\end{array}$ & $\begin{array}{l}\text { C Kristian Enestvedt } \\
\text { USA }\end{array}$ \\
\hline $\begin{array}{l}\text { Simon Coulton } \\
\text { UK }\end{array}$ & $\begin{array}{l}\text { Catherine D'este } \\
\text { Australia }\end{array}$ & $\begin{array}{l}\text { Steven Engebretson } \\
\text { USA }\end{array}$ \\
\hline $\begin{array}{l}\text { Peter Alan Coventry } \\
\text { UK }\end{array}$ & $\begin{array}{l}\text { Niveditha Devasenapathy } \\
\text { India }\end{array}$ & $\begin{array}{l}\text { Tracy Epton } \\
\text { UK }\end{array}$ \\
\hline $\begin{array}{l}\text { Peter Craig } \\
\text { UK }\end{array}$ & $\begin{array}{l}\text { Francesco Di Mola } \\
\text { Italy }\end{array}$ & $\begin{array}{l}\text { Mert Erkan } \\
\text { Germany }\end{array}$ \\
\hline $\begin{array}{l}\text { Holger Cramer } \\
\text { Germany }\end{array}$ & $\begin{array}{l}\text { Sean Dinneen } \\
\text { Ireland }\end{array}$ & $\begin{array}{l}\text { Tom Fahey } \\
\text { Ireland }\end{array}$ \\
\hline $\begin{array}{l}\text { Georgina Crichton } \\
\text { Australia }\end{array}$ & $\begin{array}{l}\text { Beate Ditzen } \\
\text { Germany }\end{array}$ & $\begin{array}{l}\text { Muhammad Faisal } \\
\text { Pakistan }\end{array}$ \\
\hline $\begin{array}{l}\text { Christine Cronin } \\
\text { USA }\end{array}$ & $\begin{array}{l}\text { Dennis Dixon } \\
\text { USA }\end{array}$ & $\begin{array}{l}\text { Anna Falanga } \\
\text { Italy }\end{array}$ \\
\hline $\begin{array}{l}\text { William Crown } \\
\text { USA }\end{array}$ & $\begin{array}{l}\text { Mary Dixon-Woods } \\
\text { UK }\end{array}$ & $\begin{array}{l}\text { Morag Farquhar } \\
\text { UK }\end{array}$ \\
\hline $\begin{array}{l}\text { Allan Cyna } \\
\text { Australia }\end{array}$ & $\begin{array}{l}\text { Peter Dodek } \\
\text { Canada }\end{array}$ & $\begin{array}{l}\text { Paul Farrand } \\
\text { UK }\end{array}$ \\
\hline
\end{tabular}




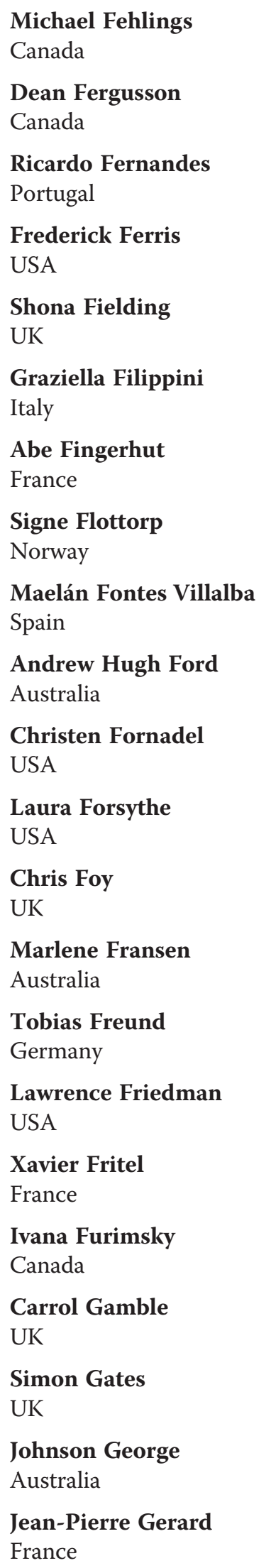

Prithwijit Ghosh

USA

Mark Gillies

Australia

Montserrat Girabent

Spain

Timothy Girard

USA

Lise Gluud

Denmark

Charlie Goldsmith

Canada

Manuel Gomes

UK

Primitivo Gómez Cardero

Spain

Mithat Gonen

USA

John Gonzalez

UK

Marjorie Good

USA

Adam Goode

USA

Jayashree Gopal

India

Jonathan Graffy

UK

Sean Grant

UK

Jocelyn Gravel

Canada

Walter Gregory

UK

Andrew Grey

New Zealand

Andrew Grieve

UK

James Griffin

UK

Xavier Griffin

UK

Ulla Griffiths

UK
Freedom Nkhululeko Gumedze

South Africa

Olivier Guyen

Switzerland

Ok Kyung Ham

Korea, South

James Hanley

Canada

Paul Harden

UK

Nicola Harman

UK

Sue Harnan

UK

Robert Hatala

Slovakia

Charles Heilig

USA

Asgeir R. Helgason

Sweden

Paul Hendrick

UK

Matthias Hermann

Switzerland

Sharlene Hesse-Biber

USA

Sarah Hetrick

Australia

Christo Heunis

South Africa

Michelle Heys

UK

Daniel Hind

UK

Christoph Hofer

Switzerland

Marie Hogan

USA

Maxi Holland

Germany

Nina Homayoon

Austria

Richard Hooper

UK 


\begin{tabular}{|c|c|c|}
\hline Val Hopwood & Lewis James & Steve Karas \\
\hline UK & UK & USA \\
\hline Dell Horey & Nicholas James & Sunil Kari \\
\hline Australia & UK & India \\
\hline $\begin{array}{l}\text { Kristina Dupont Hougaard } \\
\text { Denmark }\end{array}$ & $\begin{array}{l}\text { Murali Janakiram } \\
\text { USA }\end{array}$ & $\begin{array}{l}\text { Manuella Kaster } \\
\text { Brazil }\end{array}$ \\
\hline $\begin{array}{l}\text { Louise Michele Howard } \\
\text { UK }\end{array}$ & $\begin{array}{l}\text { Thomas Janssens } \\
\text { Belgium }\end{array}$ & $\begin{array}{l}\text { Thomas Kaulhausen } \\
\text { Germany }\end{array}$ \\
\hline $\begin{array}{l}\text { Michael Howley } \\
\text { USA }\end{array}$ & $\begin{array}{l}\text { Pawel Jastreboff } \\
\text { USA }\end{array}$ & $\begin{array}{l}\text { Walter Kernan } \\
\text { USA }\end{array}$ \\
\hline $\begin{array}{l}\text { Jui-Chien Hsieh } \\
\text { Taiwan }\end{array}$ & $\begin{array}{l}\text { Martin Jenkins } \\
\text { UK }\end{array}$ & $\begin{array}{l}\text { Sally Kerry } \\
\text { UK }\end{array}$ \\
\hline $\begin{array}{l}\text { Bo } \mathbf{H u} \\
\text { USA }\end{array}$ & $\begin{array}{l}\text { Gail Jensen } \\
\text { USA }\end{array}$ & $\begin{array}{l}\text { Line Kessel } \\
\text { Denmark }\end{array}$ \\
\hline $\begin{array}{l}\text { Dominique Hubert } \\
\text { France }\end{array}$ & $\begin{array}{l}\text { Kristin Jerger } \\
\text { USA }\end{array}$ & $\begin{array}{l}\text { David Kessler } \\
\text { UK }\end{array}$ \\
\hline $\begin{array}{l}\text { John Hummel } \\
\text { USA }\end{array}$ & $\begin{array}{l}\text { Honghua Jiang } \\
\text { USA }\end{array}$ & $\begin{array}{l}\text { Arndt Kiessling } \\
\text { Germany }\end{array}$ \\
\hline $\begin{array}{l}\text { Scott Hummel } \\
\text { USA }\end{array}$ & $\begin{array}{l}\text { Antony Johansen } \\
\text { UK }\end{array}$ & $\begin{array}{l}\text { Nanteza Gladys Kigozi } \\
\text { South Africa }\end{array}$ \\
\hline $\begin{array}{l}\text { David Hunter } \\
\text { Australia }\end{array}$ & $\begin{array}{l}\text { Robert Johansson } \\
\text { Sweden }\end{array}$ & $\begin{array}{l}\text { Seung-Nam Kim } \\
\text { USA }\end{array}$ \\
\hline $\begin{array}{l}\text { Myra Hunter } \\
\text { UK }\end{array}$ & $\begin{array}{l}\text { Kent Johnson } \\
\text { Australia }\end{array}$ & $\begin{array}{l}\text { Soo Wan Kim } \\
\text { Korea, South }\end{array}$ \\
\hline $\begin{array}{l}\text { John Hustad } \\
\text { USA }\end{array}$ & $\begin{array}{l}\text { Ashley Jones } \\
\text { UK }\end{array}$ & $\begin{array}{l}\text { Peter Kimani } \\
\text { UK }\end{array}$ \\
\hline $\begin{array}{l}\text { Peter Hutchinson } \\
\text { UK }\end{array}$ & $\begin{array}{l}\text { David Jones } \\
\text { UK }\end{array}$ & $\begin{array}{l}\text { Jonathan Kimmelman } \\
\text { Canada }\end{array}$ \\
\hline $\begin{array}{l}\text { Ana Iltis } \\
\text { USA }\end{array}$ & $\begin{array}{l}\text { Patricia Jones } \\
\text { USA }\end{array}$ & $\begin{array}{l}\text { Ann Louise Kinmonth } \\
\text { UK }\end{array}$ \\
\hline $\begin{array}{l}\text { Joanne Ingwall } \\
\text { USA }\end{array}$ & $\begin{array}{l}\text { Sue Jordan } \\
\text { UK }\end{array}$ & $\begin{array}{l}\text { Kevin Kissling } \\
\text { USA }\end{array}$ \\
\hline $\begin{array}{l}\text { George Ioannidis } \\
\text { Canada }\end{array}$ & $\begin{array}{l}\text { Lawrence Joseph } \\
\text { Canada }\end{array}$ & $\begin{array}{l}\text { Heather Kitzman-Ulrich } \\
\text { USA }\end{array}$ \\
\hline $\begin{array}{l}\text { Sameena Iqbal } \\
\text { Canada }\end{array}$ & $\begin{array}{l}\text { Andrew Judge } \\
\text { UK }\end{array}$ & $\begin{array}{l}\text { Lawrence Kleinman } \\
\text { USA }\end{array}$ \\
\hline $\begin{array}{l}\text { Khalida Ismail } \\
\text { UK }\end{array}$ & $\begin{array}{l}\text { Steven Julious } \\
\text { UK }\end{array}$ & $\begin{array}{l}\text { Roman Kloeckner } \\
\text { Germany }\end{array}$ \\
\hline $\begin{array}{l}\text { Kristina Jackson } \\
\text { USA }\end{array}$ & $\begin{array}{l}\text { Michael Kaess } \\
\text { Germany }\end{array}$ & $\begin{array}{l}\text { Sarah Knowles } \\
\text { UK }\end{array}$ \\
\hline $\begin{array}{l}\text { Adam Jacobs } \\
\text { UK }\end{array}$ & $\begin{array}{l}\text { Juliana Kain } \\
\text { Chile }\end{array}$ & $\begin{array}{l}\text { Kristen Knutson } \\
\text { USA }\end{array}$ \\
\hline $\begin{array}{l}\text { George Jallo } \\
\text { USA }\end{array}$ & $\begin{array}{l}\text { Norma Kanarek } \\
\text { USA }\end{array}$ & $\begin{array}{l}\text { Seok-Jae Ko } \\
\text { Korea, South }\end{array}$ \\
\hline
\end{tabular}


Yun Hyung Koog

Korea, South

Riikka Korja

Finland

Belchin Kostov

Spain

Helena Kraemer

USA

Michael Krams

USA

Jayashri Kulkarni

Australia

Lindsay Kuroki

USA

Sunoh Kwon

USA

Jan Kyselovic

Slovakia

Christopher Labos

Canada

Jacques Lacroix

Canada

Trudie Lang

UK

Marilyne Lange

Netherlands

Helena Länsimies-Antikainen

Finland

Marissa Lassere

Australia

Dimitrios Lathyris

Greece

Eric Lau

Hong Kong

Romy Lauche

Germany

Alexandre Lautrette

France

Julia Lawton

UK

Gerald Lebovic

Canada

Myeong Soo Lee

Korea, South
Ellen Lee

UK

Seungmin Lee

Korea, South

Stanley Lemeshow

USA

Isabel Leroux-Roels

Belgium

Stefan Leucht

Germany

Daniel Levin

USA

Beth Lewis

USA

Ruth Lewis

UK

Joel Lexchin

Canada

Ai-Hsien Li

Canada

Ai-Hsien Li

Taiwan

Shaojung Li

Taiwan

Min Li

Hong Kong

Fan-Rong Liang

China

Lalita Limaye

India

Piotr Lipiec

Poland

Jianping Liu

China

Cun-Zhi Liu

China

Teresa Liu-Ambrose

Canada

Xiong Lize

China

Francesco Locatelli

Italy

S. Scott Lollis

USA
Carl Lombard

South Africa

J Longhurst

USA

Colleen Loo

Australia

Purificación Lopez

Spain

Travis Loux

USA

Aiping Lu

Hong Kong

Jon Lurie

USA

David Lussier

Canada

Jinhui Ma

Canada

Andrew Maas

Belgium

Lynette MacKenzie

Australia

Graeme MacLennan

UK

Hugh MacPherson

UK

Michael Tvilling Madsen

Denmark

Albane B.R. Maggio

Switzerland

Tanya Mailhot

Canada

Valerio Mais

Italy

Laxmaiah Manchikanti

USA

Jun Mao

USA

Maura Marcucci

Canada

Neil Marlow

UK

Tony Marson

UK 
Arturo MartIacute-Carvajal

Venezuela

David Martin

Australia

Monica Martinez-Cengotitabengoa

Spain

Julia Mascherbauer

Austria

Karen Maschke

USA

Nancy Mayo

Canada

Lawrence Mbuagbaw

Cameroon

Amanda Mcintyre

Canada

Sharon Mckinley

Australia

Roberto Méndez-Sánchez

Spain

Hanna Merk

Sweden

Sally Merry

New Zealand

Luc Mertens

Canada

Luca Miceli

Italy

Christoph Michalski

Germany

Oliver Micke

Germany

Sandy Middleton

Australia

Jordan Miller

Canada

Vanessa Miller

USA

Jesse Mills

USA

Nicola Mills

UK

Erich Minar

Austria
Ben Mol

Afghanistan

Alan Montgomery

UK

Hayley Moore

UK

Eva Morris

UK

Tim Morris

UK

Joanna Morrison

Nepal

William Mortenson

Canada

Yoshiharu Motoo

Japan

Lawrence Moulton

USA

Brendan Mulhern

UK

Caroline Murphy

UK

Gordon Murray

UK

Jonah Musa

Nigeria

Shannon Myers Virtue

USA

Luigi Naldi

Italy

Melissa Napolitano

USA

Silvana Naredi

Sweden

Thais Nascimento

Canada

Efrat Neter

Israel

Marie-Louise Newell

UK

Tim Newton

UK

Paul Nietert

USA
Teemu Niiranen

Finland

Stavros Nikolakopoulos

Netherlands

Magnus Nilsson

Sweden

Curtis Noonan

USA

Tine Nordgreen

Norway

John Norrie

UK

Paulina Nowicka

Sweden

Xavier Nuñez

Spain

Stacy O'blenes

Canada

Alicia O'cathain

UK

Eraldo Occhetta

Italy

Lang'o Odondi

UK

Adefowope Odueyungbo

USA

Michael Okun

USA

Goran Olivecrona

Sweden

Stefano Omboni

Italy

Robert O'neill

USA

Anthony Ormerod

UK

Matthew Page

Australia

Christopher Parshuram

Canada

Nick Parsons

UK

Megan Passey

Australia 


\begin{tabular}{|c|c|c|}
\hline $\begin{array}{l}\text { James Paul } \\
\text { Canada }\end{array}$ & $\begin{array}{l}\text { Lindsay Prior } \\
\text { UK }\end{array}$ & $\begin{array}{l}\text { Rhonda Rosychuk } \\
\text { USA }\end{array}$ \\
\hline $\begin{array}{l}\text { Janet Peacock } \\
\text { UK }\end{array}$ & $\begin{array}{l}\text { Riccardo Proietti } \\
\text { Italy }\end{array}$ & $\begin{array}{l}\text { Àngel Ruiz } \\
\text { Spain }\end{array}$ \\
\hline $\begin{array}{l}\text { Nuria Perez-Alvarez } \\
\text { Spain }\end{array}$ & $\begin{array}{l}\text { Audrey Prost } \\
\text { UK }\end{array}$ & $\begin{array}{l}\text { Milind Ruke } \\
\text { India }\end{array}$ \\
\hline $\begin{array}{l}\text { Leigh Perreault } \\
\text { USA }\end{array}$ & $\begin{array}{l}\text { Eleanor Pullenayegum } \\
\text { Canada }\end{array}$ & $\begin{array}{l}\text { Roman Rukwied } \\
\text { Germany }\end{array}$ \\
\hline $\begin{array}{l}\text { Sean Perrin } \\
\text { UK }\end{array}$ & $\begin{array}{l}\text { Daryl Pullman } \\
\text { Canada }\end{array}$ & $\begin{array}{l}\text { Clare Rutterford } \\
\text { UK }\end{array}$ \\
\hline $\begin{array}{l}\text { Paolo Persichetti } \\
\text { Italy }\end{array}$ & $\begin{array}{l}\text { Helena Quesada } \\
\text { Spain }\end{array}$ & $\begin{array}{l}\text { Everardo Saad } \\
\text { Brazil }\end{array}$ \\
\hline $\begin{array}{l}\text { Christopher Phillips } \\
\text { USA }\end{array}$ & $\begin{array}{l}\text { Antoine Rabbat } \\
\text { France }\end{array}$ & $\begin{array}{l}\text { Tsuneaki Sadanaga } \\
\text { Japan }\end{array}$ \\
\hline $\begin{array}{l}\text { Sean Philpott-Jones } \\
\text { USA }\end{array}$ & $\begin{array}{l}\text { James Raftery } \\
\text { UK }\end{array}$ & $\begin{array}{l}\text { Brodie Sakakibara } \\
\text { Canada }\end{array}$ \\
\hline $\begin{array}{l}\text { Lira Pi } \\
\text { USA }\end{array}$ & $\begin{array}{l}\text { Jochen Raimann } \\
\text { USA }\end{array}$ & $\begin{array}{l}\text { Jorge Salluh } \\
\text { Brazil }\end{array}$ \\
\hline $\begin{array}{l}\text { Claude Pichonnaz } \\
\text { Switzerland }\end{array}$ & $\begin{array}{l}\text { Inderpal Randhawa } \\
\text { USA }\end{array}$ & $\begin{array}{l}\text { Zainab Samaan } \\
\text { Canada }\end{array}$ \\
\hline $\begin{array}{l}\text { Davide Pietropaoli } \\
\text { Italy }\end{array}$ & $\begin{array}{l}\text { Nicolas Rasmussen } \\
\text { Australia }\end{array}$ & $\begin{array}{l}\text { John Sampalis } \\
\text { Canada }\end{array}$ \\
\hline $\begin{array}{l}\text { Stefan Pilz } \\
\text { Austria }\end{array}$ & $\begin{array}{l}\text { Stefania Redolfi } \\
\text { France }\end{array}$ & $\begin{array}{l}\text { Peter Sandercock } \\
\text { UK }\end{array}$ \\
\hline $\begin{array}{l}\text { Carlos E. Pinfildi } \\
\text { Brazil }\end{array}$ & $\begin{array}{l}\text { Sunita Rehal } \\
\text { UK }\end{array}$ & $\begin{array}{l}\text { Carlos Santos-Gallego } \\
\text { USA }\end{array}$ \\
\hline $\begin{array}{l}\text { Paul Pinsky } \\
\text { USA }\end{array}$ & $\begin{array}{l}\text { Clare Relton } \\
\text { UK }\end{array}$ & $\begin{array}{l}\text { David Saunders } \\
\text { Thailand }\end{array}$ \\
\hline $\begin{array}{l}\text { Daniel Pinto } \\
\text { Portugal }\end{array}$ & $\begin{array}{l}\text { Matthieu Resche-Rigon } \\
\text { France }\end{array}$ & $\begin{array}{l}\text { Mary Sawtell } \\
\text { UK }\end{array}$ \\
\hline $\begin{array}{l}\text { Robert Platt } \\
\text { Canada }\end{array}$ & $\begin{array}{l}\text { Juliette Richetin } \\
\text { Italy }\end{array}$ & $\begin{array}{l}\text { Simonetta Scalvini } \\
\text { Italy }\end{array}$ \\
\hline $\begin{array}{l}\text { Jenny Ploeg } \\
\text { Canada }\end{array}$ & $\begin{array}{l}\text { Dan Riddle } \\
\text { USA }\end{array}$ & $\begin{array}{l}\text { Dirk Schadler } \\
\text { Germany }\end{array}$ \\
\hline $\begin{array}{l}\text { Gregory Pond } \\
\text { Canada }\end{array}$ & $\begin{array}{l}\text { Rachel Riechelmann } \\
\text { Brazil }\end{array}$ & $\begin{array}{l}\text { Stephen Schaffer } \\
\text { USA }\end{array}$ \\
\hline $\begin{array}{l}\text { Rudolf Poolman } \\
\text { Netherlands }\end{array}$ & $\begin{array}{l}\text { Robert Rintoul } \\
\text { UK }\end{array}$ & $\begin{array}{l}\text { Bawarjan Schatlo } \\
\text { Germany }\end{array}$ \\
\hline $\begin{array}{l}\text { Raphael Porcher } \\
\text { France }\end{array}$ & $\begin{array}{l}\text { Maria Rosaria Rizzo } \\
\text { Italy }\end{array}$ & $\begin{array}{l}\text { Roberta Scherer } \\
\text { USA }\end{array}$ \\
\hline $\begin{array}{l}\text { Martin Posch } \\
\text { Austria }\end{array}$ & $\begin{array}{l}\text { Wendy Robertson } \\
\text { UK }\end{array}$ & $\begin{array}{l}\text { Ulrike Schmidt } \\
\text { UK }\end{array}$ \\
\hline $\begin{array}{l}\text { John Powell } \\
\text { UK }\end{array}$ & $\begin{array}{l}\text { Reitze Rodseth } \\
\text { South Africa }\end{array}$ & $\begin{array}{l}\text { Simon Schneider } \\
\text { Germany }\end{array}$ \\
\hline
\end{tabular}




\begin{tabular}{|c|c|c|}
\hline $\begin{array}{l}\text { Rosa Schnyer } \\
\text { USA }\end{array}$ & $\begin{array}{l}\text { Lene Theil Skovgaard } \\
\text { Denmark }\end{array}$ & $\begin{array}{l}\text { Frank Sullivan } \\
\text { Canada }\end{array}$ \\
\hline $\begin{array}{l}\text { David Schriger } \\
\text { USA }\end{array}$ & $\begin{array}{l}\text { Susan Slaughter } \\
\text { Canada }\end{array}$ & $\begin{array}{l}\text { Qi Sun } \\
\text { China }\end{array}$ \\
\hline $\begin{array}{l}\text { Wilhelm Schulte-Mattler } \\
\text { Germany }\end{array}$ & $\begin{array}{l}\text { Martin Smalbrugge } \\
\text { Netherlands }\end{array}$ & $\begin{array}{l}\text { Chris Sutton } \\
\text { UK }\end{array}$ \\
\hline $\begin{array}{l}\text { Kenneth Schulz } \\
\text { USA }\end{array}$ & $\begin{array}{l}\text { Alexey Smetkin } \\
\text { Russian Federation }\end{array}$ & $\begin{array}{l}\text { Nao Suzuki } \\
\text { Japan }\end{array}$ \\
\hline $\begin{array}{l}\text { Julio José Secades Ruiz } \\
\text { Spain }\end{array}$ & $\begin{array}{l}\text { Helen Snooks } \\
\text { UK }\end{array}$ & $\begin{array}{l}\text { Vigdis Sveinsdottir } \\
\text { Norway }\end{array}$ \\
\hline $\begin{array}{l}\text { Sukhdev Sembi } \\
\text { UK }\end{array}$ & $\begin{array}{l}\text { Claire Snowdon } \\
\text { UK }\end{array}$ & $\begin{array}{l}\text { Matthew Robert Sydes } \\
\text { UK }\end{array}$ \\
\hline $\begin{array}{l}\text { Mehmet Sen } \\
\text { UK }\end{array}$ & $\begin{array}{l}\text { Priscilla Soares } \\
\text { Brazil }\end{array}$ & $\begin{array}{l}\text { Yutaka Takeda } \\
\text { Japan }\end{array}$ \\
\hline $\begin{array}{l}\text { Kok-Min Seow } \\
\text { Taiwan }\end{array}$ & $\begin{array}{l}\text { Mikael Sodergren } \\
\text { UK }\end{array}$ & $\begin{array}{l}\text { Monica Taljaard } \\
\text { Canada }\end{array}$ \\
\hline $\begin{array}{l}\text { Andreas Serra } \\
\text { Switzerland }\end{array}$ & $\begin{array}{l}\text { Masoud Solaymani-Dodaran } \\
\text { Iran }\end{array}$ & $\begin{array}{l}\text { Navdeep Tangri } \\
\text { Canada }\end{array}$ \\
\hline $\begin{array}{l}\text { Dean A. Sewell } \\
\text { UK }\end{array}$ & $\begin{array}{l}\text { Daniel Solomon } \\
\text { USA }\end{array}$ & $\begin{array}{l}\text { David Tappin } \\
\text { UK }\end{array}$ \\
\hline $\begin{array}{l}\text { Fabrizio Sgolastra } \\
\text { Italy }\end{array}$ & $\begin{array}{l}\text { Kjetil Soreide } \\
\text { Norway }\end{array}$ & $\begin{array}{l}\text { Warwick Teague } \\
\text { Australia }\end{array}$ \\
\hline $\begin{array}{l}\text { Dr Shalimar } \\
\text { India }\end{array}$ & $\begin{array}{l}\text { Harald Sourij } \\
\text { UK }\end{array}$ & $\begin{array}{l}\text { Melissa Teo } \\
\text { Singapore }\end{array}$ \\
\hline $\begin{array}{l}\text { Hongcai Shang } \\
\text { China }\end{array}$ & $\begin{array}{l}\text { Sonia Souto } \\
\text { Spain }\end{array}$ & $\begin{array}{l}\text { Alexandre Terrier } \\
\text { Switzerland }\end{array}$ \\
\hline $\begin{array}{l}\text { Surya Kant Sharma } \\
\text { India }\end{array}$ & $\begin{array}{l}\text { Anshu Srivastava } \\
\text { India }\end{array}$ & $\begin{array}{l}\text { Ekmel Tezel } \\
\text { Turkey }\end{array}$ \\
\hline $\begin{array}{l}\text { Anjail Sharrief } \\
\text { USA }\end{array}$ & $\begin{array}{l}\text { Cesare Stabilini } \\
\text { Italy }\end{array}$ & $\begin{array}{l}\text { George Thanassoulis } \\
\text { Canada }\end{array}$ \\
\hline $\begin{array}{l}\text { Byung-Cheul Shin } \\
\text { Korea, South }\end{array}$ & $\begin{array}{l}\text { Amanda Staiano } \\
\text { USA }\end{array}$ & $\begin{array}{l}\text { Achilleas Thoma } \\
\text { Canada }\end{array}$ \\
\hline $\begin{array}{l}\text { Joseph Patrick Shott } \\
\text { USA }\end{array}$ & $\begin{array}{l}\text { Nigel Stallard } \\
\text { UK }\end{array}$ & $\begin{array}{l}\text { Brett Thombs } \\
\text { Canada }\end{array}$ \\
\hline $\begin{array}{l}\text { Ian Shrier } \\
\text { Canada }\end{array}$ & $\begin{array}{l}\text { Simon Stanworth } \\
\text { UK }\end{array}$ & $\begin{array}{l}\text { Merran Toerien } \\
\text { UK }\end{array}$ \\
\hline $\begin{array}{l}\text { Muhammed Siddiqui } \\
\text { UK }\end{array}$ & $\begin{array}{l}\text { Emily Stockings } \\
\text { Australia }\end{array}$ & $\begin{array}{l}\text { Mattie Tops } \\
\text { Netherlands }\end{array}$ \\
\hline $\begin{array}{l}\text { Julius Sim } \\
\text { UK }\end{array}$ & $\begin{array}{l}\text { Magdalena Strach } \\
\text { Poland }\end{array}$ & $\begin{array}{l}\text { Shaun Treweek } \\
\text { UK }\end{array}$ \\
\hline $\begin{array}{l}\text { Fiona Simpson } \\
\text { Australia }\end{array}$ & $\begin{array}{l}\text { Bela Suki } \\
\text { USA }\end{array}$ & $\begin{array}{l}\text { Janette Turner } \\
\text { UK }\end{array}$ \\
\hline $\begin{array}{l}\text { Sergio Siragusa } \\
\text { Italy }\end{array}$ & $\begin{array}{l}\text { Frank Sullivan } \\
\text { UK }\end{array}$ & $\begin{array}{l}\text { Sheila Turner } \\
\text { UK }\end{array}$ \\
\hline
\end{tabular}




\begin{tabular}{|c|c|c|}
\hline $\begin{array}{l}\text { Laura Twyman } \\
\text { Australia }\end{array}$ & $\begin{array}{l}\text { Germain Weber } \\
\text { Austria }\end{array}$ & $\begin{array}{l}\text { Taixiang Wu } \\
\text { China }\end{array}$ \\
\hline $\begin{array}{l}\text { Christi Ulmer } \\
\text { USA }\end{array}$ & $\begin{array}{l}\text { Angela Webster } \\
\text { Australia }\end{array}$ & $\begin{array}{l}\text { Raphael Wurm } \\
\text { Austria }\end{array}$ \\
\hline $\begin{array}{l}\text { Thongchai Vachirarojpisan } \\
\text { Thailand }\end{array}$ & $\begin{array}{l}\text { Jacqui Webster } \\
\text { Australia }\end{array}$ & $\begin{array}{l}\text { Rudolf P. Wüthrich } \\
\text { Switzerland }\end{array}$ \\
\hline $\begin{array}{l}\text { Luc Valiquette } \\
\text { Canada }\end{array}$ & $\begin{array}{l}\text { Martin O. Weickert } \\
\text { UK }\end{array}$ & $\begin{array}{l}\text { Jielai Xia } \\
\text { China }\end{array}$ \\
\hline $\begin{array}{l}\text { Tjeerd Van Staa } \\
\text { UK }\end{array}$ & $\begin{array}{l}\text { Birgit Weinberger } \\
\text { Austria }\end{array}$ & $\begin{array}{l}\text { Lize Xiong } \\
\text { China }\end{array}$ \\
\hline $\begin{array}{l}\text { Hilde Verbeek } \\
\text { Netherlands }\end{array}$ & $\begin{array}{l}\text { Gesine Weser } \\
\text { Germany }\end{array}$ & $\begin{array}{l}\text { Bin Xu } \\
\text { China }\end{array}$ \\
\hline $\begin{array}{l}\text { Johan Verbraecken } \\
\text { Belgium }\end{array}$ & $\begin{array}{l}\text { Helen Whitford } \\
\text { Australia }\end{array}$ & $\begin{array}{l}\text { Chenglin Ye } \\
\text { Canada }\end{array}$ \\
\hline $\begin{array}{l}\text { Emily Vertosick } \\
\text { USA }\end{array}$ & $\begin{array}{l}\text { Natasha Wiebe } \\
\text { Canada }\end{array}$ & $\begin{array}{l}\text { Chiu Yen Ling } \\
\text { Taiwan }\end{array}$ \\
\hline $\begin{array}{l}\text { Roland Vetter } \\
\text { Germany }\end{array}$ & $\begin{array}{l}\text { Alishia Williams } \\
\text { Australia }\end{array}$ & $\begin{array}{l}\text { Wing-Fai Yeung } \\
\text { Hong Kong }\end{array}$ \\
\hline $\begin{array}{l}\text { Miguel Vicco } \\
\text { Argentina }\end{array}$ & $\begin{array}{l}\text { Michelle Wise } \\
\text { New Zealand }\end{array}$ & $\begin{array}{l}\text { Fang Yu } \\
\text { USA }\end{array}$ \\
\hline $\begin{array}{l}\text { Andrew Vickers } \\
\text { USA }\end{array}$ & $\begin{array}{l}\text { Claudia Witt } \\
\text { Switzerland }\end{array}$ & $\begin{array}{l}\text { Zhou Yujie } \\
\text { China }\end{array}$ \\
\hline $\begin{array}{l}\text { Marcos Vidal Melo } \\
\text { USA }\end{array}$ & $\begin{array}{l}\text { Claudia Witt } \\
\text { Germany }\end{array}$ & $\begin{array}{l}\text { Muhamad Saiful Bahri Yusoff } \\
\text { Malaysia }\end{array}$ \\
\hline $\begin{array}{l}\text { Marta Vilaró } \\
\text { Spain }\end{array}$ & $\begin{array}{l}\text { Barbara Wizner } \\
\text { Poland }\end{array}$ & $\begin{array}{l}\text { Bai-Yun Zeng } \\
\text { UK }\end{array}$ \\
\hline $\begin{array}{l}\text { Sophocles Voineskos } \\
\text { Canada }\end{array}$ & $\begin{array}{l}\text { Johannes Woitzik } \\
\text { Germany }\end{array}$ & $\begin{array}{l}\text { Qinhong Zhang } \\
\text { China }\end{array}$ \\
\hline $\begin{array}{l}\text { Julia Wade } \\
\text { UK }\end{array}$ & $\begin{array}{l}\text { Anna Wolters } \\
\text { Netherlands }\end{array}$ & $\begin{array}{l}\text { Lihui Zhao } \\
\text { USA }\end{array}$ \\
\hline $\begin{array}{l}\text { Adrian Wagg } \\
\text { Canada }\end{array}$ & $\begin{array}{l}\text { Trecia Wouldes } \\
\text { New Zealand }\end{array}$ & $\begin{array}{l}\text { Linda Zhong } \\
\text { Hong Kong }\end{array}$ \\
\hline $\begin{array}{l}\text { Rolf Wahlstrom } \\
\text { Sweden }\end{array}$ & $\begin{array}{l}\text { Darong Wu } \\
\text { China }\end{array}$ & $\begin{array}{l}\text { Bingmei Zhu } \\
\text { China }\end{array}$ \\
\hline $\begin{array}{l}\text { Stephen John Walters } \\
\text { UK }\end{array}$ & $\begin{array}{l}\text { Justin Wu } \\
\text { Hong Kong }\end{array}$ & $\begin{array}{l}\text { Merrick Zwarenstein } \\
\text { Canada }\end{array}$ \\
\hline $\begin{array}{l}\text { Shu Wang } \\
\text { China }\end{array}$ & $\begin{array}{l}\text { Yu-Tai Wu } \\
\text { USA }\end{array}$ & \\
\hline
\end{tabular}

